\title{
Hermite-Hadamard and Simpson Type Inequalities for Differentiable Quasi-Geometrically Convex Functions
}

\author{
İmdat İşcan*, Kerim Bekar, Selim Numan \\ Department of Mathematics, Faculty of Arts and Sciences, Giresun University, Giresun, Turkey \\ *Corresponding author: imdat.iscan@giresun.edu.tr \\ Received March 01, 2014; Revised April 06, 2014; Accepted April 13, 2014
}

\begin{abstract}
In this paper, the authors define a new identity for differentiable functions. By using of this identity, authors obtain new estimates on generalization of Hadamard and Simpson type inequalities for quasi-geometrically convex functions.
\end{abstract}

Keywords: quasi-geometrically convex functions, hermite-hadamard type inequalities, simpson type inequality

Cite This Article: İmdat İşcan, Kerim Bekar, and Selim Numan, "Hermite-Hadamard and Simpson Type Inequalities for Differentiable Quasi-Geometrically Convex Functions.” Turkish Journal of Analysis and Number Theory, vol. 2, no. 2 (2014): 42-46. doi: 10.12691/tjant-2-2-3.

\section{Introduction}

Let real function $f$ be defined on some nonempty interval $I$ of real line R. The function $f$ is said to be convex on $I$ if inequality

$$
f(t x+(1-t) y) \leq t f(x)+(1-t) f(y)
$$

holds for all $x, y \in I$ and $t \in[0,1]$.

Following inequalities are well known in the literature as Hermite-Hadamard inequality and Simpson inequality respectively:

Theorem 1. Let $f: I \subseteq \mathbb{R} \rightarrow \mathbb{R}$ be a convex function defined on the interval $I$ of real numbers and $a, b \in I$ with $a<b$. The following double inequality holds

$$
f\left(\frac{a+b}{2}\right) \leq \frac{1}{b-a} \int_{a}^{b} f(x) d x \leq \frac{f(a)+f(b)}{2} .
$$

Theorem 2. Let $f:[a, b] \rightarrow \mathbb{R}$ be a four times continuously differentiable mapping on $(a, b)$ and $\left\|f^{(4)}\right\|_{\infty}=\sup _{x \in(a, b)}\left|f^{(4)}(x)\right|<\infty$. Then the following inequality holds:

$$
\begin{aligned}
& \left|\frac{1}{3}\left[\frac{f(a)+f(b)}{2}+2 f\left(\frac{a+b}{2}\right)\right]-\frac{1}{b-a} \int_{a}^{b} f(x) d x\right| \\
& \leq \frac{1}{2880}\left\|f^{(4)}\right\|_{\infty}(b-a)^{4} .
\end{aligned}
$$

In recent years, many athors have studied errors estimations for Hermite-Hadamard, Ostrowski and Simpson inequalities; for refinements, counterparts, generalization see $[2,9,10]$.
The following definitions are well known in the literature.

Definition 1 ([7,8]). A function $f: I \subseteq(0, \infty) \rightarrow \mathbb{R}$ is said to be GA-convex (geometric-arithmatically convex) if

$$
f\left(x^{t} y^{1-t}\right) \leq t f(x)+(1-t) f(y)
$$

for all $x, y \in I$ and $t \in[0,1]$.

Definition 2 ([7,8]). A function $f: I \subseteq(0, \infty) \rightarrow(0, \infty)$ is said to be GG-convex (called in [13] geometrically convex function) if

$$
f\left(x^{t} y^{1-t}\right) \leq f(x)^{t} f(y)^{(1-t)}
$$

for all $x, y \in I$ and $t \in[0,1]$.

In [3], İşcan gave definition of quasi-geometrically convexity as follows:

Definition 3. A function $f: I \subseteq(0, \infty) \rightarrow \mathbb{R}$ is said to be quasi-geometrically convex on $I$ if

$$
f\left(x^{t} y^{1-t}\right) \leq \sup \{f(x), f(y)\},
$$

for any $x, y \in I$ and $t \in[0,1]$.

Clearly, any GA-convex and geometrically convex functions are quasi-geometrically convex functions. Furthermore, there exist quasi-geometrically convex functions which are neither GA-convex nor GG-convex [3].

For some recent results concerning Hermite-Hadamard type inequalities for GA-convex, GG-convex, quasigeometrically convex functions we refer interestes reader to $[1,3,4,5,6,11,12,14]$.

The goal of this article is to establish some new general integral inequalities of Hermite-Hadamard and Simpson type for quasi-geometrically convex functions by using a new integral identity. 


\section{Main Results}

Let $f: I \subseteq(0, \infty) \rightarrow \mathbb{R}$ be a differentiable function on $I^{\circ}$, the interior of $I$, throughout this section we will take

$$
\begin{aligned}
& I_{f}(\lambda, \mu, a, b)=(\lambda-\mu) f(\sqrt{a b})+\mu f(a) \\
& +(1-\lambda) f(b)-\frac{1}{\ln (b / a)} \int_{a}^{b} \frac{f(u)}{u} d u
\end{aligned}
$$

where $a, b \in I$ with $a<b$ and $\lambda, \mu \in \mathbb{R}$.

In order to prove our main results we need the following identity.

Lemma 1. Let $f: I \subseteq(0, \infty) \rightarrow \mathbb{R}$ be a differentiable function on $I^{\circ}$ such that $f^{\prime} \in L[a, b]$, where $a, b \in I$ with $a<b$. Then for all $\lambda, \mu \in \mathbb{R}$ we have:

$$
\begin{aligned}
& I_{f}(\lambda, \mu, a, b)=\ln (b / a)\left\{\int_{0}^{1 / 2}(t-\mu) a^{1-t} b^{t} f^{\prime}\left(a^{1-t} b^{t}\right) d t\right. \\
& \left.+\int_{1 / 2}^{1}(t-\lambda) a^{1-t} b^{t} f^{\prime}\left(a^{1-t} b^{t}\right) d t\right\} .
\end{aligned}
$$

Proof. By integration by parts and changing the variable, we can state

$$
\begin{aligned}
& \ln (b / a) \int_{0}^{1 / 2}(t-\mu) a^{1-t} b^{t} f^{\prime}\left(a^{1-t} b^{t}\right) d t \\
& =\int_{0}^{1 / 2}(t-\mu) d f\left(a^{1-t} b^{t}\right) \\
& =\left.(t-\mu) f\left(a^{1-t} b^{t}\right)\right|_{0} ^{1 / 2}-\int_{0}^{1 / 2} f\left(a^{1-t} b^{t}\right) d t \\
& =\left(\frac{1}{2}-\mu\right) f(\sqrt{a b})+\mu f(a)-\frac{1}{\ln (b / a)} \int_{a}^{\sqrt{a b}} \frac{f(u)}{u} d u
\end{aligned}
$$

and similarly we get

$$
\begin{aligned}
& \ln (b / a) \int_{1 / 2}^{1}(t-\lambda) a^{1-t} b^{t} f^{\prime}\left(a^{1-t} b^{t}\right) d t \\
& =\int_{1 / 2}^{1}(t-\lambda) d f\left(a^{1-t} b^{t}\right) \\
& =\left.(t-\lambda) f\left(a^{1-t} b^{t}\right)\right|_{1 / 2} ^{1}-\int_{1 / 2}^{1} f\left(a^{1-t} b^{t}\right) d t \\
& =(1-\lambda) f(b)-\left(\frac{1}{2}-\lambda\right) f(\sqrt{a b})-\frac{1}{\ln (b / a)} \int_{\sqrt{a b}}^{b} \frac{f(u)}{u} d u .
\end{aligned}
$$

Adding the resulting identities we obtain the desired result.

Theorem 3 Let $f: I \subset(0, \infty) \rightarrow \mathbb{R}$ be a differentiable function on $I^{\circ}$ such that $f^{\prime} \in L[a, b]$, where $a, b \in I^{\circ}$ with $a<b$. If $\left|f^{\prime}\right|^{q}$ is quasi-geometrically convex on $[a, b]$ for some fixed $q \geq 1$ and $0 \leq \mu \leq 1 / 2 \leq \lambda \leq 1$, then the following inequality holds

$$
\begin{aligned}
& I_{f}(\lambda, \mu, a, b) \leq \ln (b / a)\left(\sup \left\{\left|f^{\prime}(a)\right|^{q},\left|f^{\prime}(b)\right|^{q}\right\}\right)^{\frac{1}{q}} \\
& \left\{C_{1}^{1-1 / q}(\mu) C_{3}^{1 / q}(\mu, q, a, b)+C_{2}^{1-1 / q}(\lambda) C_{4}^{1 / q}(\lambda, q, a, b)\right\}
\end{aligned}
$$

where

$$
C_{1}(\mu)=\mu^{2}-\frac{\mu}{2}+\frac{1}{8},
$$

$C_{2}(\lambda)=\lambda^{2}-\frac{3 \lambda}{2}+\frac{5}{8}$,

$C_{3}(\mu, q, a, b)$

$=\left\{\begin{array}{l}\frac{1}{2 q \ln (b / a)}\left[(1-2 \mu)(a b)^{q / 2}+4 \mu a^{(1-\mu) q} L\left(a^{q \mu}, b^{q \mu}\right)\right. \\ \left.-a^{q / 2} L\left(a^{q / 2}, b^{q / 2}\right)-2 \mu a^{q}\right], 0<\mu \leq 1 / 2, \\ \frac{a^{q / 2}}{2 q \ln (b / a)}\left[b^{q / 2}-L\left(a^{q / 2}, b^{q / 2}\right)\right], \mu=0\end{array}\right.$

$C_{4}(\lambda, q, a, b)=$

$\frac{1}{2 q \ln (b / a)}\left[2(1-\lambda) b^{q}-(2 \lambda-1)(a b)^{q / 2}-2 L\left(a^{q}, b^{q}\right)\right.$

$\left.+4 \lambda a^{(1-\lambda) q} L\left(a^{q \lambda}, b^{q \lambda}\right)-a^{q / 2} L\left(a^{q / 2}, b^{q / 2}\right)\right]$,

and $L(a, b)$ is logarithmic mean defined by $L(a, b)=(b-a) /(\ln b-\ln a)$.

Proof. Since $\left|f^{\prime}\right|^{q}$ is quasi-geometrically convex on [a,b], for all $t \in[0,1]$

$$
\left|f^{\prime}\left(a^{1-t} b^{t}\right)\right|^{q} \leq \sup \left\{\left|f^{\prime}(a)\right|^{q},\left|f^{\prime}(b)\right|^{q}\right\} .
$$

Hence, using Lemma 1 and power mean inequality we get

$$
\begin{aligned}
& I_{f}(\lambda, \mu, a, b) \leq \ln (b / a) \\
& \times\left\{\left(\int_{0}^{1 / 2}|t-\mu| d t\right)^{1-\frac{1}{q}}\left(\int_{0}^{1 / 2}|t-\mu|\left(a^{1-t} b^{t}\right)^{q} \sup \left\{\left|f^{\prime}(a)\right|^{q},\left|f^{\prime}(b)\right|^{q}\right\} d t\right)^{\frac{1}{q}}\right. \\
& \left.+\left(\int_{1 / 2}^{1}|t-\lambda| d t\right)^{1-\frac{1}{q}}\left(\int_{1 / 2 \sup }^{1}|t-\lambda|\left(\left.f^{\prime}(a)\right|^{q},\left|f^{\prime}(b)\right|^{q}\right\} d t\right)^{q}\right\} \\
& \leq \ln (b / a)\left(\sup \left\{\left|f^{\prime}(a)\right|^{q},\left|f^{\prime}(b)\right|^{q}\right\}\right)^{\frac{1}{q}}
\end{aligned}
$$

$$
\begin{aligned}
& \times\left\{\left(\int_{0}^{1 / 2}|t-\mu| d t\right)^{1-\frac{1}{q}}\left(\int_{0}^{1 / 2}|t-\mu|\left(a^{1-t} b^{t}\right)^{q} d t\right)^{\frac{1}{q}}\right. \\
& \left.+\left(\int_{1 / 2}^{1}|t-\lambda| d t\right)^{1-\frac{1}{q}}\left(\int_{1 / 2}^{1}|t-\lambda|\left(a^{1-t} b^{t}\right)^{q} d t\right)^{\frac{1}{q}}\right\},
\end{aligned}
$$


where

$$
\begin{aligned}
& \int_{0}^{1 / 2}|t-\mu| d t=C_{1}(\mu)=\mu^{2}-\frac{\mu}{2}+\frac{1}{8}, \\
& \int_{1 / 2}^{1}|t-\lambda| d t=C_{2}(\lambda)=\lambda^{2}-\frac{3 \lambda}{2}+\frac{5}{8}, \\
& \int_{0}^{1 / 2}|t-\mu|\left(a^{1-t} b^{t}\right)^{q} d t=C_{3}(\mu, q, a, b), \\
& \int_{1 / 2}^{1}|t-\lambda|\left(a^{1-t} b^{t}\right)^{q} d t=C_{4}(\lambda, q, a, b),
\end{aligned}
$$

which completes the proof.

Corollary 1 Under the assumptions of Theorem 3 with $\lambda=\mu=1 / 2$, the inequality (2) reduced to the following inequality

$$
\begin{aligned}
& \left|\frac{f(a)+f(b)}{2}-\frac{1}{\ln (b / a)} \int_{a}^{b} \frac{f(u)}{u} d u\right| \\
& \leq\left(\frac{1}{8}\right)^{1-1 / q} \ln (b / a)\left(\sup \left\{\begin{array}{l}
\left|f^{\prime}(a)\right|^{q}, \\
\left|f^{\prime}(b)\right|^{q}
\end{array}\right\}\right)^{\frac{1}{q}}\left\{C_{3}^{1 / q}(1 / 2, q, a, b)\right. \\
& \leq\left(\frac{1}{8}\right)^{1-1 / q} \ln (b / a)\left(\sup \left\{\begin{array}{l}
\left|f^{\prime}(a)\right|^{q}, \\
\left|f^{\prime}(b)\right|^{q}
\end{array}\right\}\right)^{\frac{1}{q}} \\
& \left.\times\left\{C_{3}^{1 / q}(0, q, a, b)+C_{4}^{1 / q}(1, q, a, b)\right\}+C_{4}^{1 / q}(1 / 2, q, a, b)\right\} .
\end{aligned}
$$

Corollary 2 Under the assumptions of Theorem 3 with $\mu=0$ and $\lambda=1$, the inequality (2) reduced to the following inequality

$$
\begin{aligned}
& \left|f(\sqrt{a b})-\frac{1}{\ln (b / a)} \int_{a}^{b} \frac{f(u)}{u} d u\right| \\
& \leq\left(\frac{1}{8}\right)^{1-1 / q} \ln (b / a)\left(\sup \left\{\left|f^{\prime}(a)\right|^{q},\left|f^{\prime}(b)\right|^{q}\right\}\right)^{\frac{1}{q}} \\
& \times\left\{C_{3}^{1 / q}(0, q, a, b)+C_{4}^{1 / q}(1, q, a, b)\right\} .
\end{aligned}
$$

Corollary 3 Under the assumptions of Theorem 3 with $\mu=1 / 6$ and $\lambda=5 / 6$, the inequality (2) reduced to the following inequality

$$
\begin{aligned}
& \left|\frac{1}{3}\left[\frac{f(a)+f(b)}{2}+2 f(\sqrt{a b})\right]-\frac{1}{\ln (b / a)} \int_{a}^{b} \frac{f(u)}{u} d u\right| \\
& \left.\leq\left(\frac{5}{72}\right)^{1-1 / q} \ln (b / a)\left(\sup \left\{\begin{array}{l}
\left|f^{\prime}(a)\right|^{q}, \\
\left|f^{\prime}(b)\right|^{q}
\end{array}\right\}\right)\right)^{\frac{1}{q}} \\
& \times\left\{\begin{array}{l}
C_{3}^{1 / q}(1 / 6, q, a, b) \\
+C_{4}^{1 / q}(5 / 6, q, a, b)
\end{array}\right\}
\end{aligned}
$$

Theorem 4 Let $f: I \subset(0, \infty) \rightarrow \mathbb{R}$ be a differentiable function on $I^{\circ}$ such that $f^{\prime} \in L[a, b]$, where $a, b \in I^{\circ}$ with $a<b$. If $\left|f^{\prime}\right|^{q}$ is quasi-geometrically convex on [a,b] for some fixed $q>1$ and $0 \leq \mu \leq 1 / 2 \leq \lambda \leq 1$, then the following inequality holds.

$$
\begin{aligned}
& I_{f}(\lambda, \mu, a, b) \leq \ln (b / a)\left(\sup \left\{\left|f^{\prime}(a)\right|^{q},\left|f^{\prime}(b)\right|^{q}\right\}\right)^{\frac{1}{q}} \\
& \times\left\{C_{5}^{1 / p}(p, \mu) C_{7}^{1 / q}(q, a, b)+C_{6}^{1 / p}(p, \lambda) C_{8}^{1 / q}(q, a, b)\right\}
\end{aligned}
$$

where

$$
\begin{aligned}
& C_{5}(p, \mu)=\frac{1}{p+1}\left[\mu^{p+1}+\left(\frac{1}{2}-\mu\right)^{p+1}\right], \\
& C_{6}(p, \lambda)=\frac{1}{p+1}\left[\left(\lambda-\frac{1}{2}\right)^{p+1}+(1-\lambda)^{p+1}\right], \\
& C_{7}(q, a, b)=\frac{1}{2} a^{q / 2} L\left(a^{q / 2}, b^{q / 2}\right), \\
& C_{8}(q, a, b)=L\left(a^{q}, b^{q}\right)-C_{7}(q, a, b)
\end{aligned}
$$

and $\frac{1}{p}+\frac{1}{q}=1$.

Proof. Since $\left|f^{\prime}\right|^{q}$ is quasi-geometrically convex on $[a, b]$ and using Lemma 1 and Hölder inequality, we get

$$
\begin{aligned}
& I_{f}(\lambda, \mu, a, b) \leq \ln (b / a) \\
& \times\left\{\left(\int_{0}^{1 / 2}|t-\mu|^{p} d t\right)^{\frac{1}{p}}\left(\int_{0}^{1 / 2}\left(a^{1-t} b^{t}\right)^{q} \sup \left\{\begin{array}{l}
\left|f^{\prime}(a)\right|^{q}, \\
\left|f^{\prime}(b)\right|^{q}
\end{array}\right\} d t\right)^{\frac{1}{q}}\right. \\
& \left.+\left(\int_{1 / 2}^{1}|t-\lambda|^{p} d t\right)^{\frac{1}{p}}\left(\int_{1 / 2}^{1}\left(a^{1-t} b^{t}\right)^{q} \sup \left\{\begin{array}{l}
\left|f^{\prime}(a)\right|^{q}, \\
\left|f^{\prime}(b)\right|^{q}
\end{array}\right\} d t\right)^{\frac{1}{q}}\right\} \\
& \leq \ln (b / a)\left(\sup \left\{\left|f^{\prime}(a)\right|^{q},\left|f^{\prime}(b)\right|^{q}\right\}\right)^{\frac{1}{q}} \\
& \times\left\{\left(\int_{0}^{1 / 2}|t-\mu|^{p} d t\right)^{\frac{1}{p}}\left(\int_{0}^{1 / 2}\left(a^{1-t} b^{t}\right)^{q} d t\right)^{\frac{1}{q}}\right. \\
& \left.+\left(\int_{1 / 2}^{1}|t-\lambda|^{p} d t\right)^{\frac{1}{p}}\left(\int_{1 / 2}^{1}\left(a^{1-t} b^{t}\right)^{q} d t\right)^{\frac{1}{q}}\right\},
\end{aligned}
$$

here it is seen by simple computation that

$$
\begin{aligned}
& \int_{0}^{1 / 2}|t-\mu|^{p} d t=\frac{1}{p+1}\left[\mu^{p+1}+\left(\frac{1}{2}-\mu\right)^{p+1}\right], \\
& \int_{1 / 2}^{1}|t-\lambda|^{p} d t=\frac{1}{p+1}\left[\left(\lambda-\frac{1}{2}\right)^{p+1}+(1-\lambda)^{p+1}\right], \\
& \int_{0}^{1 / 2}\left(a^{1-t} b^{t}\right)^{q} d t=\frac{a^{q / 2}}{2} L\left(a^{q / 2}, b^{q / 2}\right) \\
& \text { and } \int_{1 / 2}^{1}\left(a^{1-t} b^{t}\right)^{q} d t=L\left(a^{q}, b^{q}\right)-\frac{a^{q / 2}}{2} L\left(a^{q / 2}, b^{q / 2}\right) .
\end{aligned}
$$


Hence, the proof is completed.

Corollary 4 Under the assumptions of Theorem 4 with $\lambda=\mu=1 / 2$, the inequality (4) reduced to the following inequality

$$
\begin{aligned}
& \left|\frac{f(a)+f(b)}{2}-\frac{1}{\ln (b / a)} \int_{a}^{b} \frac{f(u)}{u} d u\right| \\
& \leq \ln (b / a)\left(\sup \left\{\left|f^{\prime}(a)\right|^{q},\left|f^{\prime}(b)\right|^{q}\right\}\right)^{\frac{1}{q}} \\
& \times\left(\frac{1}{2^{p+1}(p+1)}\right)^{1 / p}\left\{C_{7}^{1 / q}(q, a, b)+C_{8}^{1 / q}(q, a, b)\right\} .
\end{aligned}
$$

Corollary 5 Under the assumptions of Theorem 4 with $\mu=0$ and $\lambda=1$, the inequality (4) reduced to the following inequality.

$$
\begin{aligned}
& \left|f(\sqrt{a b})-\frac{1}{\ln (b / a)} \int_{a}^{b} \frac{f(u)}{u} d u\right| \\
& \leq \ln (b / a)\left(\sup \left\{\left|f^{\prime}(a)\right|^{q},\left|f^{\prime}(b)\right|^{q}\right\}\right)^{\frac{1}{q}} \\
& \times\left(\frac{1}{2^{p+1}(p+1)}\right)^{1 / p}\left\{C_{7}^{1 / q}(q, a, b)+C_{8}^{1 / q}(q, a, b)\right\} .
\end{aligned}
$$

Corollary 6 Under the assumptions of Theorem 4 with $\mu=1 / 6$ and $\lambda=5 / 6$, the inequality (4) reduced to the following inequality

$$
\begin{aligned}
& \left|\frac{1}{3}\left[\frac{f(a)+f(b)}{2}+2 f(\sqrt{a b})\right]-\frac{1}{\ln (b / a)} \int_{a}^{b} \frac{f(u)}{u} d u\right| \\
& \leq \frac{\ln (b / a)}{2}\left(\sup \left\{\left|f^{\prime}(a)\right|^{q},\left|f^{\prime}(b)\right|^{q}\right\}\right)^{\frac{1}{q}} \\
& \times\left(\frac{1+2^{p+1}}{6^{p+1}(p+1)}\right)^{1 / p}\left\{C_{7}^{1 / q}(q, a, b)+C_{8}^{1 / q}(q, a, b)\right\} .
\end{aligned}
$$

Theorem 5 Let $f: I \subset(0, \infty) \rightarrow \mathbb{R}$ be a differentiable function on $I^{\circ}$ such that $f^{\prime} \in L[a, b]$, where $a, b \in I^{\circ}$ with $a<b$. If $\left|f^{\prime}\right|^{q}$ is quasi-geometrically convex on $[a, b]$ for some fixed $q>1$ and $0 \leq \mu \leq 1 / 2 \leq \lambda \leq 1$, then the following inequality holds

$$
\begin{aligned}
& I_{f}(\lambda, \mu, a, b) \\
& \left.\leq \ln (b / a)\left(\sup \left\{\begin{array}{l}
\left|f^{\prime}(a)\right|^{q}, \\
\left|f^{\prime}(b)\right|^{q}
\end{array}\right\}\right)\right)^{\frac{1}{q}} \\
& \times\left\{C_{7}^{1 / p}(p, a, b) C_{5}^{1 / q}(q, \mu)+C_{8}^{1 / p}(p, a, b) C_{6}^{1 / q}(q, \lambda)\right\}
\end{aligned}
$$

where $C_{5}, C_{6}, C_{7}, C_{8}$ are defined as in Theorem 4 and $\frac{1}{p}+\frac{1}{q}=1$
Proof. Since $\left|f^{\prime}\right|^{q}$ is quasi-geometrically convex on $[a, b]$ and using Lemma 1 and Hölder inequality, we get

$$
\begin{aligned}
& I_{f}(\lambda, \mu, a, b) \leq \ln (b / a) \\
& \times\left\{\left(\int_{0}^{1 / 2}\left(a^{1-t} b^{t}\right)^{p} d t\right)^{\frac{1}{p}}\left(\int_{0}^{1 / 2}|t-\mu|^{q} \sup \left\{\begin{array}{l}
\left|f^{\prime}(a)\right|^{q}, \\
\left|f^{\prime}(b)\right|^{q}
\end{array}\right\} d t\right)^{\frac{1}{q}}\right. \\
& \left.+\left(\int_{1 / 2}^{1}\left(a^{1-t} b^{t}\right)^{p} d t\right)^{\frac{1}{p}}\left(\int_{1 / 2}^{1}|t-\lambda|^{q} \sup \left\{\begin{array}{l}
\left|f^{\prime}(a)\right|^{q}, \\
\left|f^{\prime}(b)\right|^{q}
\end{array}\right\} d t\right)^{\frac{1}{q}}\right\} \\
& \leq \ln (b / a)\left(\sup \left\{\left|f^{\prime}(a)\right|^{q},\left|f^{\prime}(b)\right|^{q}\right\}\right)^{\frac{1}{q}} \\
& \left\{\left(\int_{0}^{1 / 2}\left(a^{1-t} b^{t}\right)^{p} d t\right)^{\frac{1}{p}}\left(\int_{0}^{1 / 2}|t-\mu|^{q} d t\right)^{\frac{1}{q}}\right. \\
& \left.+\left(\int_{1 / 2}^{1}\left(a^{1-t} b^{t}\right)^{p} d t\right)^{\frac{1}{p}}\left(\int_{1 / 2}^{1}|t-\lambda|^{q} d t\right)^{\frac{1}{q}}\right\}, \\
& \leq \ln (b / a)\left(\sup \left\{\left|f^{\prime}(a)\right|^{q},\left|f^{\prime}(b)\right|^{q}\right\}\right)^{\frac{1}{q}} \\
& \left\{C_{7}^{1 / p}(p, a, b) C_{5}^{1 / q}(q, \mu)+C_{8}^{1 / p}(p, a, b) C_{6}^{1 / q}(q, \lambda)\right\} .
\end{aligned}
$$

Hence, the proof is completed.

Corollary 7 Under the assumptions of Theorem 5 with $\lambda=\mu=1 / 2$, the inequality (5) reduced to the following inequality

$$
\begin{aligned}
& \left|\frac{f(a)+f(b)}{2}-\frac{1}{\ln (b / a)} \int_{a}^{b} \frac{f(u)}{u} d u\right| \\
& \leq \ln (b / a)\left(\sup \left\{\left|f^{\prime}(a)\right|^{q},\left|f^{\prime}(b)\right|^{q}\right\}\right)^{\frac{1}{q}} \\
& \times\left(\frac{1}{2^{q+1}(q+1)}\right)^{1 / q}\left\{C_{7}^{1 / p}(p, a, b)+C_{8}^{1 / p}(p, a, b)\right\} .
\end{aligned}
$$

Corollary 8 Under the assumptions of Theorem 5 with $\mu=0$ and $\lambda=1$, the inequality (5) reduced to the following inequality

$$
\begin{aligned}
& \left|f(\sqrt{a b})-\frac{1}{\ln (b / a)} \int_{a}^{b} \frac{f(u)}{u} d u\right| \\
& \leq \ln (b / a)\left(\sup \left\{\left|f^{\prime}(a)\right|^{q},\left|f^{\prime}(b)\right|^{q}\right\}\right)^{\frac{1}{q}} \\
& \times\left(\frac{1}{2^{q+1}(q+1)}\right)^{1 / q}\left\{C_{7}^{1 / p}(p, a, b)+C_{8}^{1 / p}(p, a, b)\right\} .
\end{aligned}
$$


Corollary 9 Under the assumptions of Theorem 5 with $\mu=1 / 6$ and $\lambda=5 / 6$, the inequality (5) reduced to the following inequality

$$
\begin{aligned}
& \left|\frac{1}{3}\left[\frac{f(a)+f(b)}{2}+2 f(\sqrt{a b})\right]-\frac{1}{\ln (b / a)} \int_{a}^{b} \frac{f(u)}{u} d u\right| \\
& \leq \frac{\ln (b / a)}{2}\left(\sup \left\{\left|f^{\prime}(a)\right|^{q},\left|f^{\prime}(b)\right|^{q}\right\}\right)^{\frac{1}{q}} \\
& \times\left(\frac{1+2^{q+1}}{6^{q+1}(q+1)}\right)^{1 / q}\left\{C_{7}^{1 / p}(p, a, b)+C_{8}^{1 / p}(p, a, b)\right\} .
\end{aligned}
$$

\section{References}

[1] Hua, J., Xi, B.-Y. and Qi, F., "Hermite-Hadamard type inequalities for Geometric-arithmetically S-convex functions", Commun. Korean Math. Soc., 29 (1). 51-63. 2014.

[2] İşcan, İ.,"Generalization of different type integral inequalities for $S$-convex functions via fractional integrals", Applicable Analysis, 2013.

[3] İșcan, İ., "New general integral inequalities for quasigeometrically convex functions via fractional integrals", Journal of Inequalities and Applications, 2013. 491. 15 pages. 2013.
[4] İşcan, İ., "Some New Hermite-Hadamard Type Inequalities for Geometrically Convex Functions", Mathematics and Statistics, 1 (2). 86-91. 2013.

[5] İşcan, İ., "Some Generalized Hermite-Hadamard Type Inequalities for Quasi-Geometrically Convex Functions", American Journal of Mathematical Analysis, 1 (3). 48-52. 2013.

[6] İşcan, İ., "Hermite-Hadamard type inequalities for GA-S-convex functions", Le Matematiche, 2014a. Accepted for publication.

[7] Niculescu, C. P., "Convexity according to the geometric mean", Math. Inequal. Appl., 3 (2). 155-167. 2000.

[8] Niculescu, C. P., "Convexity according to means", Math. Inequal. Appl., 6 (4). 571-579. 2003.

[9] Sarıkaya, M. Z. and Aktan, N., "On the generalization of some integral inequalities and their applications", Math. Comput. Modelling, 54. 2175-2182. 2011.

[10] Sarıkaya, M. Z., Set, E. and Ozdemir, M. E., "On new inequalities of Simpson's type for S-convex functions", Comput. Math. Appl. 60. 2191-2199. 2010.

[11] Shuang, Y., Yin, H.-P. and Qi, F., "Hermite-Hadamard type integral inequalities for geometric-arithmetically S-convex functions", Analysis (Munich), 33 (2). 197-208. 2013.

[12] Zhang, X.-M., Chu, Y.-M. and Zhang, X.-H. "The HermiteHadamard Type Inequality of GA-Convex Functions and Its Application", Journal of Inequalities and Applications. 2010. Article ID 507560. 11 pages. 2010.

[13] Zhang, T.-Y., Ji, A.-P. and Qi, F., "On Integral Inequalities of Hermite-Hadamard Type for S-Geometrically Convex Functions", Abstr. Appl. Anal., 2012. Article ID 560586. 14 pages. 2012.

[14] Zhang, T.-Y., Ji, A.-P. and Qi, F., "Some inequalities of HermiteHadamard type for GA-convex functions with applications to means", Le Matematiche, LXVIII- Fasc. I. 229-239. 2013. 